\title{
Robust integral sliding mode control design for stability enhancement of under-actuated quadcopter
}

\author{
Safeer Ullah, Adeel Mehmood, Qudrat Khan, Sakhi Rehman, and Jamshed Iqbal*
}

\begin{abstract}
In this paper, a robust backstepping integral sliding mode control (RBISMC) technique is designed for the flight control of a quadcopter, which is an under-actuated nonlinear system. First, the mathematical model of this highly coupled and under-actuated system is described in the presence of dissipative drag forces. Second, a robust control algorithm is designed for the derived model to accurately track the desired outputs while ensuring the stability of attitude, altitude and position of the quadcopter. A step by step mathematical analysis, based on the Lyapunov stability theory, is performed that endorses the stability of both the fully-actuated and under-actuated subsystems of the aforementioned model. The comparison of proposed RBISMC control algorithm, with fraction order integral sliding mode control (FOISMC), affirms the enhanced performance in terms of faster states convergence, improved chattering free tracking and more robustness against uncertainties in the system.
\end{abstract}

Keywords: Unmanned aerial vehicle, quadcopter, under-actuated, backstepping, integral sliding mode control.

\section{INTRODUCTION}

The quadcopter, a special case of Unmanned Ariel Vehicles (UAV), has distinctive characteristics of flying in nearly all types of weather condition. These vehicles have attenuating ability against the undesirable torques produced by the reverse rotation of propellers installed on the opposite sides. High degrees of maneuverability enable them to hover with enhanced payload capacity and faster mobility [1]. Their longitude, altitude and attitude are controlled by four contra-rotating propellers. The quadcopter has a wide range of applications in defense, military, media, agriculture and other industries including but not limited to; mining, oil and gas, logistics and transportation. The dynamic model of the aforesaid aerial vehicle is inherently known as an under-actuated for the fact that they have lesser number of control inputs than the degrees of freedom. Therefore, the control design of such a system is a very challenging task [2]. In this regards, a lot of work has been conducted on the control design and implementation of the quadcopter. Some relevant work is mentioned here. Proportional Integral Derivative (PID) control is the most widely used linear control scheme in the industry. For example, in [3], a gain-scheduled PID was applied for the reference tracking of both transnational and rotational position of a quadcopter. The response time observed minor overshoot whereas the steady-state error was nearly equal to zero. Cowling et al. [4] applied linear quadratic regulator (LQR) controller to achieve an exact reference tracking in simulation even in the presence of disturbances. However, PID and LQR based control techniques have some performance limitations when applied to the quadcopter with unmodeled system's dynamics [5]. In [6], a nonlinear technique i.e., integral backstepping was used to control the angular rotations and linear translations of quadcopter in the presence of aerodynamic effects. Shi et al. [7] presented the design of fractional-order backstepping SMC for the control of attitude and altitude of the OS4 quadcopter. Their project provided the capability to hover and collision avoidance.It is evident that the order reduction in SMC algorithm provides the insensitivity to matched disturbance (i.e., matched disturbance rejection) [8], nevertheless, the high-frequency oscillations issue may degrade the performance of system [9]. This problem is overcame via different advanced higher-order SMC techniques (see for instance [10-12]). However, the key performance factor i.e. robustness was unfortunately lost while alleviating the problem of high-frequency oscillations. In the existing literature, some differentiators were used to overcome the unwanted effects of uncertainties and provide a precise estimation of the outputs' derivatives of the system $[13,14]$. Apart from this, ma-

Safeer Ullah and Adeel Mehmood are with the Department of Electrical \& Computer Engineering, COMSATS University, Islamabad, Pakistan (e-mail: engrxamir@gmail.com, adeel.mehmood@comsats.edu.pk). Qudrat Khan is with Center for Advanced Studies in Telecommunication, COMSATS University, Islamabad, Pakistan (e-mail: qudratullah@comsats.edu.pk). Sakhi Rehman is with Electrical Engineering Department, University of Science and Technology, Bannu, Pakistan (e-mail: engr.sakhi@gmail.com). Jamshed Iqbal is with the Department of Electrical and Electronics Engineering, University of Jeddah, Saudi Arabia and Department of Electrical Engineering, FAST National University of Computer and Emerging Sciences, Islamabad, Pakistan (e-mail: iqbal.jam@gmail.com).

* Corresponding author. 
jority of the nonlinear systems may result in undesirable behavior of the reaching phase of SMC (and even in the instability of the system's dynamics) [15]. Therefore, a reaching-phase free ISMC scheme was presented in [16] that provided both the chattering reduction and robustness improvement. It is also worthy to mention that [16] only controlled the altitude subsystem that is not much sufficient for the full flight maneuver of a quadcopter. Consequently, the above literature discussions motivate to propose a nonlinear control scheme that must provide the full flight control of a quadcopter with reduced chattering and improved robustness simultaneously.

In this paper, a synthesized approach of backstepping and integral sliding mode strategies is presented for a highly nonlinear and complex under-actuated quadcopter system. The main control task is tracking the system on the desired trajectories. In other words, the system's output $(x, y, z, \varphi, \vartheta, \psi)$ are needed to track the desired trajectories for both the fully-actuated and under-actuated control loops of the quadcopter system. The main contributions in this article are three fold. At first, the overall model of the quadcopter with under-actuated and fullyactuated loops is considered. Secondly, we have utilized the good features of the two nonlinear control techniques i.e., (in case of backstepping) easy nonlinear control design for each state and (in case of sliding modes) the invariance property. Moreover, instead of conventional SMC, an ISMC approach is used to enhance the robustness from the very start of the process i.e., invariance property is ensured from the initial time instant which never happens in case of conventional SMC. In addition, the unwanted chattering phenomena is also alleviated by the use of a strong reachability law. Finally, the proposed RBISMC is compared with the standard control strategy i.e., FOISMC presented in [17]. The result comparison demonstrates that the proposed control scheme is more appealing in term of regulation and trajectory tracking. The rest of the paper is organized as follows: In Section 2, the mathematical model of a quadcopter system is presented. The RBISMC nonlinear control strategy is derived in Section 3. This section also includes the simulations and discussions. Finally, Section 4 concludes the paper.

\section{MATHEMATICAL MODEL OF QUADCOPTER}

Mathematical model of the aforementioned system is essential for the design of control law which must be capable enough to ensures the closed loop stability. Various modeling based control strategies for this system have been discussed recently by many researchers, as described previously. This section describes the rigid body dynamics, kinematics of fixed and body reference frames and forces applied to it. The quadcopter system has three translational states $(x, y, z)$, three rotational states $(\varphi, \vartheta, \psi)$ and their derivatives $(\dot{x}, \dot{y}, \dot{z}, \dot{\varphi}, \dot{\vartheta}, \dot{\psi})$ as shown in Figure 1.

The rotational motion represents the mathematical expression of roll $\varphi$, pitch $\vartheta$ and yaw $\psi$ while the translational motion is the combination of position in the horizontal plane $(x, y)$ and altitude $z$. The quadcopter's motion is considered as a rigid body motion which is described by inertial earth frame $[X, Y, Z]$ and a fixed body frame $[x, y, z]$. The explicit expression of the rotational motion dynamics [18], in terms of angular positions and velocities, are expressed as follows

$$
\left.\begin{array}{l}
\ddot{\varphi}=\frac{\mathcal{I}_{R} \dot{\vartheta} \bar{\omega}}{\mathcal{I}_{x}}+\frac{\left(\mathcal{I}_{y}-\mathcal{I}_{z}\right)}{\mathcal{I}_{x}} \dot{\vartheta} \dot{\psi}+\frac{b l}{\mathcal{I}_{x}} \mathcal{U}_{2}-\frac{\mathcal{K}_{1}}{\mathcal{I}_{x}}\|\dot{\varphi}\|^{2} \\
\ddot{\vartheta}=\frac{-\mathcal{I}_{R} \dot{\varphi} \bar{\omega}}{\mathcal{I}_{y}}+\frac{\left(\mathcal{I}_{z}-\mathcal{I}_{x}\right)}{\mathcal{I}_{y}} \dot{\varphi} \dot{\psi}+\frac{b l}{\mathcal{I}_{y}} \mathcal{U}_{3}-\frac{\mathcal{K}_{2}}{\mathcal{I}_{y}}\|\dot{\vartheta}\|^{2} \\
\ddot{\psi}=\frac{\left(\mathcal{I}_{x}-\mathcal{I}_{y}\right)}{\mathcal{I}_{z}} \dot{\varphi} \dot{\vartheta}+\frac{d}{\mathcal{I}_{z}} \mathcal{U}_{4}-\frac{\mathcal{K}_{3}}{\mathcal{I}_{z}}\|\dot{\psi}\|^{2}
\end{array}\right\}
$$

where $b$ is the thrust coefficient, $d$ is the drag factor, $\mathcal{I}_{R}$ is the rotor inertia, $l$ is the arm's length, $\bar{\omega}=\omega_{1}+\omega_{3}-\omega_{2}-$ $\omega_{4}$ is an aerodynamic disturbance, $\omega_{i}(i=1,2,3,4)$ represents the angular speed of each brush-less DC motor, $\mathcal{K}_{1}$, $\mathcal{K}_{2}, \mathcal{K}_{3}$ are the coefficients of aero-dynamic frictions, $\mathcal{U}_{2}=$ $-\omega_{2}^{2}+\omega_{4}^{2}, \mathcal{U}_{3}=\omega_{1}^{2}-\omega_{3}^{2}$ and $\mathcal{U}_{4}=-\omega_{1}^{2}+\omega_{2}^{2}-\omega_{3}^{2}+\omega_{4}^{2}$ are the thrust produced by the respective motors which are required for the motion along $\varphi, \vartheta$ and $\psi$, respectively.

Now, by following the Euler-Newtonian equations of motion [18], one can represent the translational motion by the following equations

$$
\begin{aligned}
& \ddot{x}=(\sin \psi \sin \varphi+\cos \psi \sin \vartheta \cos \varphi) \frac{b}{m} \mathcal{U}_{1}-\frac{\overline{\mathcal{K}}_{d_{x}}}{m}\|\dot{x}\|^{2} \\
& \ddot{y}=(\sin \psi \sin \vartheta \cos \varphi-\cos \psi \sin \varphi) \frac{b}{m} \mathcal{U}_{1}-\frac{\overline{\mathcal{K}}_{d_{y}}}{m}\|\dot{y}\|^{2} \\
& \ddot{z}=(\cos \vartheta \cos \varphi) \frac{b}{m} \mathcal{U}_{1}-g-\frac{\overline{\mathcal{K}}_{d_{z}}}{m}\|\dot{z}\|^{2}
\end{aligned}
$$

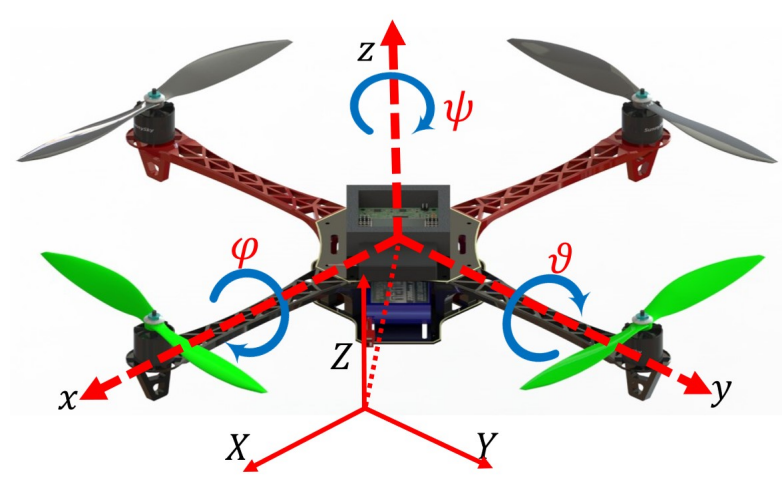

Fig. 1. The inertial and body frames of a quadcopter

where $g$ is the gravitational acceleration, $m$ is mass of the quadcopter, $b$ is the thrust factor of the propeller and 
$\mathcal{U}_{1}$ is the control input for the translational motion generation. The drag force constant is defined by $\overline{\mathcal{K}}_{d}=\rho \mathcal{A} \mathcal{K}_{d}$, where $\rho$ is mass density of the airflow that depends on the height above the sea level, $\mathcal{A}$ is the specific area of the quadcopter, $\dot{x}, \dot{y}$ and $\dot{z}$ are the speeds of translational axis in space relative to the air flow and $\mathcal{K}_{d}$ is the coefficient of air drag force and it depends on the structure of body.

Now, the overall mathematical model of a quadcopter system, in state space, can be described as follows

$$
\left.\begin{array}{l}
\dot{z}_{1}=z_{2}, \quad \dot{z}_{2}=\left(\cos \vartheta_{1} \cos \varphi_{1}\right) b_{0} \mathcal{U}_{1}+\Delta_{z}-g-\mathcal{K}_{z} z_{2}^{2}, \\
\dot{\psi}_{1}=\psi_{2}, \quad \dot{\psi}_{2}=a_{5} \vartheta_{2} \varphi_{2}+b_{3} \mathcal{U}_{4}+\Delta_{\psi}-\mathcal{K}_{\psi} \psi_{2}^{2}
\end{array}\right\}
$$

where $u_{x}=(\sin \psi \sin \varphi+\cos \psi \sin \vartheta \cos \varphi), \quad u_{y}=$ $(\sin \psi \sin \vartheta \cos \varphi-\cos \psi \sin \varphi), b_{0}=\frac{b}{m}, b_{1}=\frac{l}{\mathcal{I}_{x}}, b_{2}=$ $\frac{l}{\mathcal{I}_{y}}, b_{3}=\frac{1}{\mathcal{I}_{z}}, a_{1}=\frac{\left(\mathcal{I}_{y}-\mathcal{I}_{z}\right)}{\mathcal{I}_{x}}, a_{2}=\frac{\mathcal{I}_{R}}{\mathcal{I}_{x}}, a_{3}=\frac{\left(\mathcal{I}_{z}-\mathcal{I}_{x}\right)}{\mathcal{I}_{y}}, a_{4}=$ $\frac{-\mathcal{I}_{R}}{\mathcal{I}_{y}}, a_{5}=\frac{\left(\mathcal{I}_{x}-\mathcal{I}_{y}\right)}{\mathcal{I}_{z}}, \mathcal{K}_{(x, y, z)}=\frac{\rho \mathcal{A} \mathcal{K}_{d}}{m}, \mathcal{K}_{(\varphi, \vartheta, \psi)}=\frac{\mathcal{K}_{(1,2,3)}}{\mathcal{I}_{(x, y, z)}}$. In addition, $\Delta_{z}$ and $\Delta_{\psi}$ are the matched uncertainties.

Assumption 1: The uncertainty terms $\Delta_{z}$ and $\Delta_{\psi}$ are assumed to be matched and norm bounded by their respective positive constants $\lambda_{z}$ and $\lambda_{\psi}$ i.e., $\left|\Delta_{z}\right| \leq \lambda_{z}$ and $\left|\Delta_{\psi}\right| \leq \lambda_{\psi}$

The system parameters, used in the simulation, are given in table 1 .

Table 1. Parameters of quadcopter system

\begin{tabular}{|c|c|c|c|}
\hline Parameter & Symbol & Value & Unit \\
\hline Quadcopter mass & $m$ & 0.650 & Kg \\
\hline \multirow{2}{*}{ Inertia constants } & $\mathcal{I}_{x}=\mathcal{I}_{y}$ & $7.5 \times 10^{-3}$ & \multirow{2}{*}{$\mathrm{kg} . \mathrm{m}^{2}$} \\
\cline { 2 - 3 } & $\mathcal{I}_{z}$ & $1.3 \times 10^{-2}$ & \\
\hline Thrust coeff. & $b$ & $3.13 \times 10^{-5}$ & \multirow{2}{*}{$\mathrm{N} . \mathrm{s}^{2}$} \\
\hline Drag factor & $d$ & $7.5 \times 10^{-7}$ & \multirow{2}{*}{$\mathrm{N} . \mathrm{m}^{2} \mathrm{~s}^{2}$} \\
\hline Rotor inertia & $\mathcal{I}_{r}$ & $6 \times 10^{-5}$ & \multirow{2}{*}{$\mathrm{kg} . \mathrm{m}^{2}$} \\
\hline Arm length & $l$ & 0.23 & $\mathrm{~m}$ \\
\hline \multirow{2}{*}{ Drag coeff. } & $\mathcal{K}_{x}=\mathcal{K}_{y}$ & $5.56 \times 10^{-4}$ & \multirow{2}{*}{$\mathrm{N} / \mathrm{m} / \mathrm{s}$} \\
\cline { 2 - 3 } & $\mathcal{K}_{z}$ & $6.35 \times 10^{-4}$ & \\
\hline \multirow{2}{*}{ Aero coeff. } & $\mathcal{K}_{\varphi}=\mathcal{K}_{\vartheta}$ & $5.56 \times 10^{-4}$ & \multirow{2}{*}{$\mathrm{N} / \mathrm{rad} / \mathrm{s}$} \\
\cline { 2 - 3 } & $\mathcal{K}_{\psi}$ & $6.35 \times 10^{-4}$ & \\
\hline
\end{tabular}

It is worthy to mention that for the ease of control law design, the proposed control law is derived by dividing the system model into two subsystems i.e., the fully-actuated subsystem and the under-actuated subsystem. In the fullyactuated subsystem, the number of outputs $(z, \psi)$ are equal to the number of control inputs $\left(U_{1}, U_{4}\right)$, whereas in the under-actuated subsystem, the number of control inputs
$U_{2}$ and $U_{3}$ are less than the number of outputs $(x, y, \varphi$, $\vartheta)$. The main objective of this work is to follow a full flight desired trajectory by the quadcopter in the presence of matched uncertainties. For this purpose, an RBISMC control strategy is presented in section 3 .

\section{CONTROL DESIGN}

Since the quadcopter is an under-actuated system with strong coupling effect among the rotors' inputs, therefore, a nonlinear RBISMC scheme is proposed to achieve the stability of both fully-actuated and under-actuated subsystems.

\subsection{Fully-actuated subsystem}

The error between the reference and actual trajectory serve as control variable in the controller, which in turn generates the control inputs. The control inputs, when applied to the actuator, vary the speed of the relative motors. Consequently, the desired motion of a quadcopter is achieved. For this purpose, the reference tracking errors are defined as follow

$$
\left.\begin{array}{rll}
\varepsilon_{z_{1}}=z_{1}-z_{d} & \Rightarrow & \dot{\varepsilon}_{z_{1}}=z_{2}-\dot{z}_{d} \\
\varepsilon_{\psi_{1}}=\psi_{1}-\psi_{d} & \Rightarrow & \dot{\varepsilon}_{\psi_{1}}=\psi_{2}-\dot{\psi}_{d}
\end{array}\right\}
$$

where $z_{d}$ and $\psi_{d}$ are the reference outputs. By taking the derivatives of Lyapunov candidate functions $V_{z_{1}}=\frac{1}{2} \varepsilon_{z_{1}}^{2}$ and $V_{\psi_{1}}=\frac{1}{2} \varepsilon_{\psi_{1}}^{2}$ along (3b) and (4), one has

$$
\left.\begin{array}{ccc}
\dot{V}_{z_{1}}=\varepsilon_{z_{1}} \dot{\varepsilon}_{z_{1}} & \Rightarrow & \dot{V}_{z_{1}}=\varepsilon_{z_{1}}\left(z_{2}-\dot{z}_{d}\right) \\
\dot{V}_{\psi_{1}}=\varepsilon_{\psi_{1}} \dot{\varepsilon}_{\psi_{1}} & \Rightarrow & \dot{V}_{\psi_{1}}=\varepsilon_{\psi_{1}}\left(\psi_{2}-\dot{\psi}_{d}\right)
\end{array}\right\}
$$

Now, the variables $z_{2}$ and $\psi_{2}$ are treated as virtual control inputs in such a way that they will ensure the derivative of Lyapunov function negative definite. Thus, the following choices lead us

$$
z_{2}^{\star}=\dot{z}_{d}-\kappa_{z_{1}} \varepsilon_{z_{1}}, \quad \psi_{2}^{\star}=\dot{\psi}_{d}-\kappa_{\psi_{1}} \varepsilon_{\psi_{1}}
$$

By substituting (7) in (5), one has

$$
\dot{V}_{z_{1}}=-\kappa_{z_{1}} \varepsilon_{z_{1}}^{2}, \quad \dot{V}_{\psi_{1}}=-\kappa_{\psi_{1}} \varepsilon_{\psi_{1}}^{2}
$$

where $\kappa_{z_{1}}$ and $\kappa_{\psi_{1}}$ are the positive design constants. Now, proceeding toward the next step, we will consider the aforementioned $z_{2}^{*}$ and $\psi_{2}^{\star}$ (in (6)) as new modified references for the state $z_{2}$ and $\psi_{2}$ in the second step

$$
z_{2}^{\star}=\varepsilon_{z_{2}}+\dot{z}_{d}-\kappa_{z_{1}} \varepsilon_{z_{1}}, \quad \psi_{2}^{\star}=\varepsilon_{\psi_{2}}+\dot{\psi}_{d}-\kappa_{\psi_{1}} \varepsilon_{\psi_{1}}
$$

where $\varepsilon_{z_{2}}=z_{2}-z_{2}^{\star}$ and $\varepsilon_{\psi_{2}}=\psi_{2}-\psi_{2}^{\star}$.

At the second step, the interest is that $z_{2}$ should track $z_{2}^{*}$ and, similarly, $\psi_{2}$ should track $\psi_{2}^{\star}$. In order to meet these requirements, integral manifolds of the following form are designed

$$
\beta_{z_{2}}=z_{2}-z_{2}^{\star}+\mathfrak{z}_{z}, \quad \beta_{\psi_{2}}=\psi_{2}-\psi_{2}^{\star}+\mathfrak{z} \psi
$$

where $\mathfrak{z}_{z}=\gamma_{z} \int \varepsilon_{z_{2}} d t, \mathfrak{z}_{\psi}=\gamma_{\psi} \int \varepsilon_{\psi_{2}} d t$ and $\gamma_{z}$ and $\gamma_{\psi}$ are the positive constants. 
Remark 1: At this stage, the objective of the proposed control scheme is to drive the outputs $\left(z_{2}, \psi_{2}\right)$ of the fullyactuated subsystem on the desired trajectory, which is possible by steering their sliding manifolds to zero in finite time with the help of control inputs $\mathcal{U}_{1}=\mathcal{U}_{10}+\mathcal{U}_{11}$ and $\mathcal{U}_{4}=\mathcal{U}_{40}+\mathcal{U}_{41}$. Note that $\mathcal{U}_{10}$ and $\mathcal{U}_{40}$, are the continuous control components which are designed via pole placement method. These components governs the system dynamics in sliding mode whereas the other components $\mathcal{U}_{11}$ and $\mathcal{U}_{41}$ are designed by integral sliding mode approach such that these components establish sliding mode from the very beginning. The design of these control components, for their respective dynamics, is presented in the forthcoming theorem.

Theorem 1: Consider that the fully-actuated subsystem (3b) remains true subject to the Assumption 1. If the backstepping procedure based virtual control laws $z_{2}^{\star}$ and $\psi_{2}^{\star}$ and the integral sliding manifolds are chosen according to (7) and (8), respectively, then the following control laws will ensure finite time sliding mode enforcement and consequently asymptotic convergence of the errors states.

$$
\begin{aligned}
\mathcal{U}_{11}= & \frac{1}{b_{0}\left(\cos \vartheta_{1} \cos \varphi_{1}\right)}\left(g+\mathcal{K}_{z} z_{2}^{2}+\dot{z}_{2}^{*}-\kappa_{z_{2}} \beta_{z_{2}}\right. \\
& \left.-\kappa_{z_{3}} \operatorname{sign}\left(\beta_{z_{2}}\right)\right) \\
\mathcal{U}_{41}= & \frac{1}{b_{3}}\left(-a_{5} \vartheta_{2} \varphi_{2}+\mathcal{K}_{\psi} \psi_{2}^{2}+\dot{\psi}_{2}^{*}-\kappa_{\psi_{2}} \beta_{\psi_{2}}\right. \\
& \left.-\kappa_{\psi_{3}} \operatorname{sign}\left(\beta_{\psi_{2}}\right)\right)
\end{aligned}
$$

where $\kappa_{z_{2}}, \kappa_{z_{3}}, \kappa_{\psi_{2}}$ and $\kappa_{\psi_{3}}$ are the positive design constants.

Proof. To prove this theorem, consider the time derivative of sliding manifolds $\beta_{z_{2}}$ and $\beta_{\psi_{2}}$ (represented in (9)) along (3b), one obtains

$$
\left.\begin{array}{c}
\dot{\beta}_{z_{2}}=\cos \vartheta_{1} \cos \varphi_{1} b_{0} \mathcal{U}_{1}+\Delta_{z}-g-\mathcal{K}_{z} z_{2}^{2}-\dot{z}_{2}^{*}+\dot{\mathfrak{z}}_{z} \\
\dot{\beta}_{\psi_{2}}=a_{5} \vartheta_{2} \varphi_{2}+b_{3} \mathcal{U}_{4}+\Delta_{\psi}-\mathcal{K}_{\psi} \psi_{2}^{2}-\dot{\psi}_{2}^{*}+\dot{\mathfrak{z}}_{\psi}
\end{array}\right\}
$$

The following choice of the integral dynamics $\dot{\mathfrak{z}}_{z}$ and $\dot{\mathfrak{z}}_{\psi}$, with positive constants $\rho_{z}, \rho_{\psi}$

$$
\begin{gathered}
\dot{\mathfrak{z}}_{z}=-\cos \vartheta_{1} \cos \varphi_{1} b_{0} \mathcal{U}_{10} \text { with } \mathcal{U}_{10}=-\rho_{z}\left(z_{2}-z_{2}^{\star}\right) \\
\dot{\mathfrak{z}}_{\psi}=-b_{3} \mathcal{U}_{40} \text { with } \mathcal{U}_{40}=-\rho_{\psi}\left(\psi_{2}-\psi_{2}^{\star}\right)
\end{gathered}
$$

reduces (10) to the following forms

$$
\left.\begin{array}{l}
\dot{\beta}_{z_{2}}=\cos \vartheta_{1} \cos \varphi_{1} b_{0} \mathcal{U}_{11}+\Delta_{z}-g-\mathcal{K}_{z} z_{2}^{2}-\dot{z}_{2}^{*} \\
\dot{\beta}_{\psi_{2}}=a_{5} \vartheta_{2} \varphi_{2}+b_{3} \mathcal{U}_{41}+\Delta_{\psi}-\mathcal{K}_{\psi} \psi_{2}^{2}-\dot{\psi}_{2}^{*}
\end{array}\right\}
$$

Now, consider the time derivatives of extended Lyapunov functions $V_{z_{2}}=V_{z_{1}}+\frac{1}{2} \beta_{z_{2}}^{2}$ and $V_{\psi_{2}}=V_{\psi_{1}}+\frac{1}{2} \beta_{\psi_{2}}^{2}$ along 12 , one may get

$$
\begin{aligned}
\dot{V}_{z_{2}} & =\varepsilon_{z_{1}} \varepsilon_{z_{2}}-\kappa_{z_{1}} \varepsilon_{z_{1}}^{2}-\kappa_{z_{2}} \beta_{z_{2}}^{2}+\beta_{z_{2}}\left(\Delta_{z}-\kappa_{z_{3}} \operatorname{sign}\left(\beta_{z_{2}}\right)\right) \\
& \leq \varepsilon_{z_{1}} \varepsilon_{z_{2}}-\kappa_{z_{1}} \varepsilon_{z_{1}}^{2}-\kappa_{z_{2}} \beta_{z_{2}}^{2}-\left(\kappa_{z_{3}}-\left|\Delta_{z}\right|\right)\left|\beta_{z_{2}}\right| \\
\dot{V}_{z_{2}} & \leq \varepsilon_{z_{1}} \varepsilon_{z_{2}}-\kappa_{z_{1}} \varepsilon_{z_{1}}^{2}-\kappa_{z_{2}} \beta_{z_{2}}^{2}-\eta_{z}\left|\beta_{z_{2}}\right| \\
\dot{V}_{\psi_{2}} & =\varepsilon_{\psi_{1}} \varepsilon_{\psi_{2}}-\kappa_{\psi_{1}} \varepsilon_{\psi_{1}}^{2}-\kappa_{\psi_{2}} \beta_{\psi_{2}}^{2}+\left(\Delta_{\psi}-\kappa_{\psi_{3}} \operatorname{sign}\left(\beta_{\psi_{2}}\right)\right) \beta_{\psi_{2}} \\
& \leq \varepsilon_{\psi_{1}} \varepsilon_{\psi_{2}}-\kappa_{\psi_{1}} \varepsilon_{\psi_{1}}^{2}-\kappa_{\psi_{2}} \beta_{\psi_{2}}^{2}-\left(\kappa_{\psi_{3}}-\left|\Delta_{\psi}\right|\right)\left|\beta_{\psi_{2}}\right| \\
\dot{V}_{\psi_{2}} & \leq \varepsilon_{\psi_{1}} \varepsilon_{\psi_{2}}-\kappa_{\psi_{1}} \varepsilon_{\psi_{1}}^{2}-\kappa_{\psi_{2}} \beta_{\psi_{2}}^{2}-\eta_{\psi}\left|\beta_{\psi_{2}}\right|
\end{aligned}
$$

These inequalities (13) remain true only if $\kappa_{z_{3}}-\left|\Delta_{z}\right| \geq \eta_{z}$ and $\kappa_{\psi_{3}}-\left|\Delta_{\psi}\right| \geq \eta_{\psi}$, where $\eta_{z}$ and $\eta_{\psi}$ are small positive numbers. The control components $U_{10}$ and $U_{40}$ are used to steer their respective mismatches $\varepsilon_{z_{2}}$ and $\varepsilon_{\psi_{2}}$ to zero, which result in vanishing of the terms $\varepsilon_{z_{1}} \varepsilon_{z_{2}}$ and $\varepsilon_{\psi_{1}} \varepsilon_{\psi_{2}}$ (see [19] for more detail). Consequently, it confirms the negative definiteness of the augmented Lyapunov functions $\dot{V}_{z_{2}}$ and $\dot{V}_{\psi_{2}}$. Now, moving a step back, the backstepping based virtual controllers $z_{2}^{\star}$ and $\psi_{2}^{\star}$ ensured the enforcement of $z_{1}$ and $\psi_{1}$ to $z_{d}$ and $\psi_{d}$, respectively. Hence, this results in the asymptotic convergence of the states of the fully-actuated subsystem of the quadcopter to their desired references. Similarly, convergence holds for the inequalities (13).

\subsection{Under-actuated subsystem}

Now, for the ease of control design, the model of an under-actuated subsystem ( $3 b$ ) can be re-written in the form

$$
\left.\begin{array}{rl}
\dot{\chi}_{1}=\chi_{2} ; & \left.\dot{\chi}_{2}=\digamma_{1}+\right\rceil_{1} \Omega_{1} \\
\dot{\theta}_{1}=\theta_{2} ; & \left.\dot{\theta}_{2}=\digamma_{2}+\right\rceil_{2} \Omega_{2}
\end{array}\right\}
$$

where $\chi_{1}=\left[\begin{array}{l}x_{1} \\ y_{1}\end{array}\right], \chi_{2}=\left[\begin{array}{l}x_{2} \\ y_{2}\end{array}\right], \digamma_{1}=\left[\begin{array}{l}-\mathcal{K}_{x} x_{2}^{2} \\ -\mathcal{K}_{y} y_{2}^{2}\end{array}\right], \Omega_{1}=\left[\begin{array}{l}u_{x} \\ u_{y}\end{array}\right]$, \urcorner$_{1}=b_{0} \mathcal{U}_{1}\left[\begin{array}{ll}1 & 0 \\ 0 & 1\end{array}\right], \theta_{1}=\left[\begin{array}{l}\varphi_{1} \\ \vartheta_{1}\end{array}\right], \Omega_{2}=\left[\begin{array}{l}\mathcal{U}_{2} \\ \mathcal{U}_{3}\end{array}\right], \theta_{2}=\left[\begin{array}{l}\varphi_{2} \\ \vartheta_{2}\end{array}\right]$, $\digamma_{2}=\left[\begin{array}{ll}a_{1} \psi_{2} \vartheta_{2}+a_{2} \bar{\omega} & \vartheta_{2}-\mathcal{K}_{\varphi} \varphi_{2}^{2} \\ a_{3} \psi_{2} \varphi_{2}+a_{4} \bar{\omega} & \varphi_{2}-\mathcal{K}_{\vartheta} \vartheta_{2}^{2}\end{array}\right]$ and $\rceil_{2}=\left[\begin{array}{cc}b_{1} & 0 \\ 0 & b_{2}\end{array}\right]$.

The reference tracking errors between the desired reference and actual outputs are defined as

$$
\left.\begin{array}{lll}
\varepsilon_{\chi_{1}}=\chi_{1}-\chi_{d} & \Rightarrow & \dot{\varepsilon}_{\chi_{1}}=\chi_{2}-\dot{\chi}_{d} \\
\varepsilon_{\theta_{1}}=\theta_{1}-\theta_{d} & \Rightarrow & \dot{\varepsilon}_{\theta_{1}}=\theta_{2}-\dot{\theta}_{d}
\end{array}\right\}
$$

where $\chi_{d}=\left[\begin{array}{ll}x_{d} & y_{d}\end{array}\right]^{T}$ and $\theta_{d}=\left[\begin{array}{ll}\varphi_{d} & \vartheta_{d}\end{array}\right]^{T}$ are the reference outputs. The derivatives of Lyapunov functions $\left(V_{\chi_{1}}=\frac{1}{2} \varepsilon_{\chi_{1}}^{2}\right)$ and $V_{\theta_{1}}=\frac{1}{2} \varepsilon_{\theta_{1}}^{2}$ along (14) is defined as

$$
\left.\begin{array}{lll}
\dot{V}_{\chi_{1}}=\varepsilon_{\chi_{1}} \dot{\varepsilon}_{\chi_{1}} & \Rightarrow & \dot{V}_{\chi_{1}}=\varepsilon_{\chi_{1}}\left(\chi_{2}-\dot{\chi}_{d}\right) \\
\dot{V}_{\theta_{1}}=\varepsilon_{\theta_{1}} \dot{\varepsilon}_{\theta_{1}} & \Rightarrow \quad & \dot{V}_{\theta_{1}}=\varepsilon_{\theta_{1}}\left(\theta_{2}-\dot{\theta}_{d}\right)
\end{array}\right\}
$$

where the variables $\chi_{2}$ and $\theta_{2}$ are chosen as virtual control inputs that will ensure the stability of $\chi_{1}$ and $\theta_{1}$. Hence, the virtual control inputs will be

$$
\chi_{2}^{\star}=\dot{\chi}_{d}-\kappa_{\chi_{1}} \varepsilon_{\chi_{1}}, \quad \theta_{2}^{\star}=\dot{\theta}_{d}-\kappa_{\theta_{1}} \varepsilon_{\theta_{1}}
$$


where $\kappa_{\chi_{1}}$ and $\kappa_{\theta_{1}}$ are the positive design constants. By substituting (19) in (16), one gets

$$
\dot{V}_{\chi_{1}}=-\kappa_{\chi_{1}} \varepsilon_{\chi_{1}}^{2}, \quad \dot{V}_{\theta_{1}}=-\kappa_{\theta_{1}} \varepsilon_{\theta_{1}}^{2}
$$

Now, proceeding towards the last step, we will consider the following $\chi_{2}^{*}$ and $\theta_{2}^{\star}$ (in (17)) as new modified desired references for the state $\chi_{2}$ and $\theta_{2}$ in the form

$$
\chi_{2}^{\star}=\varepsilon_{\chi_{2}}+\dot{\chi}_{d}-\kappa_{\chi_{1}} \varepsilon_{\chi_{1}}, \quad \theta_{2}^{\star}=\varepsilon_{\theta_{2}}+\dot{\theta}_{d}-\kappa_{\theta_{1}} \varepsilon_{\theta_{1}}
$$

where $\varepsilon_{\chi_{2}}=\chi_{2}-\chi_{2}^{\star}$ and $\varepsilon_{\theta_{2}}=\theta_{2}-\theta_{2}^{\star}$. Now, to ensure the convergence of $\varepsilon_{\chi_{2}}$ and $\varepsilon_{\theta_{2}}$, the desired sliding surfaces $\beta_{\chi_{2}}$ and $\beta_{\theta_{2}}$ will be,

$$
\beta_{\chi_{2}}=\chi_{2}-\chi_{2}^{\star}+\mathfrak{z} \chi, \quad \beta_{\theta_{2}}=\theta_{2}-\theta_{2}^{\star}+\mathfrak{z} \theta
$$

where $\mathfrak{z}_{\chi}=\gamma_{\chi} \int \varepsilon_{\chi_{2}} d t$ and $\mathfrak{z}_{\theta}=\gamma_{\theta} \int \varepsilon_{\theta_{2}} d t$ and $\gamma_{\chi}$ and $\gamma_{\theta}$ are the positive constants.

Theorem 2: Consider that the dynamics of underactuated subsystem (in (3b)) are transformed into (14). If the backstepping procedure based virtual control laws $\chi_{2}^{\star}$ and $\theta_{2}^{\star}$ and the integral sliding manifolds are chosen according to (19) and (20), respectively, then the following control laws will ensure finite-time sliding mode enforcement and consequently asymptotic convergence of the errors states.

$$
\left.\begin{array}{l}
\Omega_{11}=\frac{1}{\urcorner_{1}}\left(\dot{\chi}_{2}^{*}-\digamma_{1}-\kappa_{\chi_{2}} \beta_{\chi_{2}}-\kappa_{\chi_{3}} \operatorname{sign}\left(\beta_{\chi_{2}}\right)\right) \\
\Omega_{21}=\frac{1}{7_{2}}\left(\dot{\theta}_{2}^{*}-\digamma_{2}-\kappa_{\theta_{2}} \beta_{\theta_{2}}-\kappa_{\theta_{3}} \operatorname{sign}\left(\beta_{\theta_{2}}\right)\right)
\end{array}\right\}
$$

Proof. To proceed to the proof, consider the time derivative of integral manifolds $\left(\beta_{\chi_{2}}, \beta_{\theta_{2}}\right.$ in (20)) along (14), one has

$$
\left.\begin{array}{l}
\left.\dot{\beta}_{\chi_{2}}=\digamma_{1}+\right\rceil_{1} \Omega_{11}-\chi_{2}^{*}+\dot{\mathfrak{z}}_{\chi} \\
\left.\dot{\beta}_{\theta_{2}}=\digamma_{2}+\right\rceil_{2} \Omega_{21}-\theta_{2}^{*}+\dot{\mathfrak{z}}_{\theta}
\end{array}\right\}
$$

The derivative of the integral dynamics $\dot{\mathfrak{z}}_{\chi}$ and $\dot{\mathfrak{z}}_{\theta}$, with positive constants $\rho_{\chi}$ and $\rho_{\theta}$, can be chosen as

$$
\left.\begin{array}{ll}
\left.\dot{\mathfrak{z}}_{\chi}=-\right\urcorner_{1} \Omega_{10} & \text { with } \quad \Omega_{10}=-\rho_{\chi}\left(\chi_{2}-\chi_{2}^{\star}\right) \\
\left.\dot{\mathfrak{z}}_{\theta}=-\right\urcorner_{2} \Omega_{20} & \text { with } \quad \Omega_{20}=-\rho_{\theta}\left(\theta_{2}-\theta_{2}^{\star}\right)
\end{array}\right\}
$$

to reduce equation (22) to the following forms

$$
\left.\left.\dot{\beta}_{\chi_{2}}=\digamma_{1}+\right\rceil_{1} \Omega_{11}-\dot{\chi}_{2}^{*}, \dot{\beta}_{\theta_{2}}=\digamma_{2}+\right\rceil_{2} \Omega_{21}-\dot{\theta}_{2}^{*}
$$

Now, to prove the stability of the aforesaid subsystem, the time derivatives of the augmented Lyapunov functions $V_{\chi_{2}}=V_{\chi_{1}}+\frac{1}{2} \beta_{\chi_{2}}^{2}$ and $V_{\theta_{2}}=V_{\theta_{1}}+\frac{1}{2} \beta_{\theta_{2}}^{2}$ along (18) and (24) are as follow

$$
\left.\begin{array}{rl}
\dot{V}_{\chi_{2}} & =\varepsilon_{\chi_{1}} \varepsilon_{\chi_{2}}-\kappa_{\chi_{1}} \varepsilon_{\chi_{1}}^{2}-\kappa_{\chi_{2}} \beta_{\chi_{2}}^{2}-\kappa_{\chi_{3}} \beta_{\chi_{2}} \operatorname{sign}\left(\beta_{\chi_{2}}\right) \\
& \leq \varepsilon_{\chi_{1}} \varepsilon_{\chi_{2}}-\kappa_{\chi_{1}} \varepsilon_{\chi_{1}}^{2}-\kappa_{\chi_{2}} \beta_{\chi_{2}}^{2}-\kappa_{\chi_{3}}\left|\beta_{\chi_{2}}\right| \\
\dot{V}_{\theta_{2}} & =\varepsilon_{\theta_{1}} \varepsilon_{\theta_{2}}-\kappa_{\theta_{1}} \varepsilon_{\theta_{1}}^{2}-\kappa_{\theta_{2}} \beta_{\theta_{2}}^{2}-\kappa_{\theta_{3}} \beta_{\theta_{2}} \operatorname{sign}\left(\beta_{\theta_{2}}\right) \\
& \leq \varepsilon_{\theta_{1}} \varepsilon_{\theta_{2}}-\kappa_{\theta_{1}} \varepsilon_{\theta_{1}}^{2}-\kappa_{\theta_{2}} \beta_{\theta_{2}}^{2}-\kappa_{\theta_{3}}\left|\beta_{\theta_{2}}\right|
\end{array}\right\}
$$

Once again, by a proper choice of these constants, one may get the negative definite $\dot{V}_{\chi_{2}}$ and $\dot{V}_{\theta_{2}}$. Consequently, the asymptotic convergence of $\varepsilon_{\chi_{2}}$ and $\varepsilon_{\theta_{2}}$ to zero is ensured. In similar fashion, the convergence of $\chi_{1}$ and $\theta_{1}$ to the desired $\chi_{d}$ and $\theta_{d}$ is also ensured via backstepping based virtual control laws $\theta_{2}^{\star}$ and $\chi_{2}^{\star}$.

Thus, taking into account equations (14) and (21), the applied control inputs for under-actuated subsystem can be expressed by the following forms

$$
\left.\begin{array}{rl}
u_{x_{1}}= & \frac{1}{b_{0} \mathcal{U}_{1}}\left(\mathcal{K}_{x} x_{2}^{2}+\dot{x}_{2}^{*}-\kappa_{x_{2}} \beta_{x_{2}}-\kappa_{x_{3}} \operatorname{sign}\left(\beta_{x_{2}}\right)\right) \\
u_{y_{1}}= & \frac{1}{b_{0} \mathcal{U}_{1}}\left(\mathcal{K}_{y} y_{2}^{2}+\dot{y}_{2}^{*}-\kappa_{y_{2}} \beta_{y_{2}}-\kappa_{y_{3}} \operatorname{sign}\left(\beta_{y_{2}}\right)\right) \\
\mathcal{U}_{21}= & \frac{1}{b_{1}}\left(\mathcal{K}_{\varphi} \varphi_{2}^{2}-a_{1} \psi_{2} \vartheta_{2}-a_{2} \bar{\omega} \vartheta_{2}+\dot{\varphi}_{2}^{*}\right. \\
& \left.-\kappa_{\varphi_{2}} \beta_{\varphi_{2}}-\kappa_{\varphi_{3}} \operatorname{sign}\left(\beta_{\varphi_{2}}\right)\right) \\
\mathcal{U}_{31}= & \frac{1}{b_{2}}\left(\mathcal{K}_{\vartheta} \vartheta_{2}^{2}-a_{3} \psi_{2} \varphi_{2}-a_{4} \bar{\omega} \varphi_{2}+\dot{\vartheta}_{2}^{*}\right. \\
& \left.-\kappa_{\vartheta_{2}} \beta_{\vartheta_{2}}-\kappa_{\vartheta_{3}} \operatorname{sign}\left(\beta_{\vartheta_{2}}\right)\right)
\end{array}\right\}
$$

The desired Euler angles $\varphi_{d}, \vartheta_{d}$ and $\psi_{d}$ will be obtained from the basic trigonometry of quadcopter (as shown in figure 1). By following the defined expression of [20], the desired $\varphi_{d}, \vartheta_{d}$ and $\psi_{d}$ can be obtained with the following expressions

$$
\left.\begin{array}{l}
\varphi_{d}=\sin ^{-1}\left(u_{x} \sin \psi_{1}-u_{y} \cos \psi_{1}\right) \\
\vartheta_{d}=\sin ^{-1}\left(\frac{u_{x} \cos \psi_{d}-u_{y} \sin \psi_{d}}{\cos \varphi_{d}}\right) \\
\psi_{d}=\tan ^{-1}\left(\frac{y_{d}-y_{1}}{x_{d}-x_{1}}\right)
\end{array}\right\}
$$

where $u_{x}$ and $u_{y}$ are obtained using the proposed control scheme (26).

In the next subsection, the simulation results of the quadcopter based on RBISMC scheme are presented.

\subsection{Simulation Results}

In this section, the proposed RBISMC control scheme has been simulated to control the dynamic model of quadcopter in MATLAB/Simulink environment in the presence of matched uncertainties. The behavior of the aforementioned control scheme (which is shown in figure 2) is compared with the FOISMC technique available in the literature [17].

In figure 2 , the $(\mathcal{U}-$ to $-\omega)$ conversion block shows the conversion of applied forces/torques $\left(\mathcal{U}_{1}, \mathcal{U}_{2}, \mathcal{U}_{3}, \mathcal{U}_{4}\right)$ into the motor speeds $\left(\omega_{1}, \omega_{2}, \omega_{3}, \omega_{4}\right)$. When the angular speeds of both the right and left propellers are increased (or decreased) and that of back and front propellers are decreased (or increased), then the motion along yaw angle " $\psi$ " is produced. Similarly, if the thrust (produced by the 
four propellers rotating at same angular speed $\omega_{i}$ ) is equal to the quadcopter's weight then it will counterbalance the acceleration due to gravity in a hover condition. In addition, the forces/torques (required for the stable flight of the quadcopter in all feasible conditions) are delivered by the high thrust propellers. Note that the high torque servos and gears are used to achieve the desired angular speed of the brush-less DC motors.

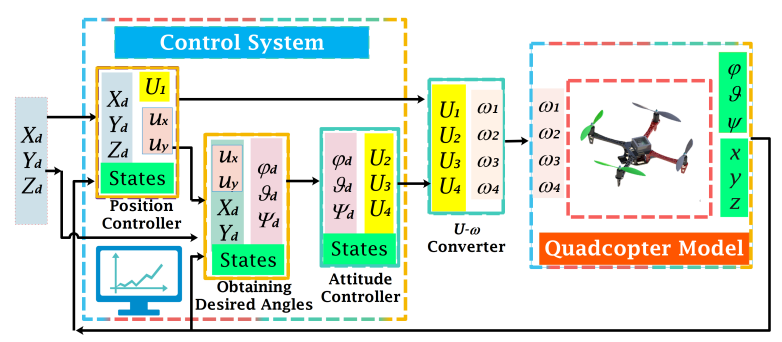

Fig. 2. Generic control system
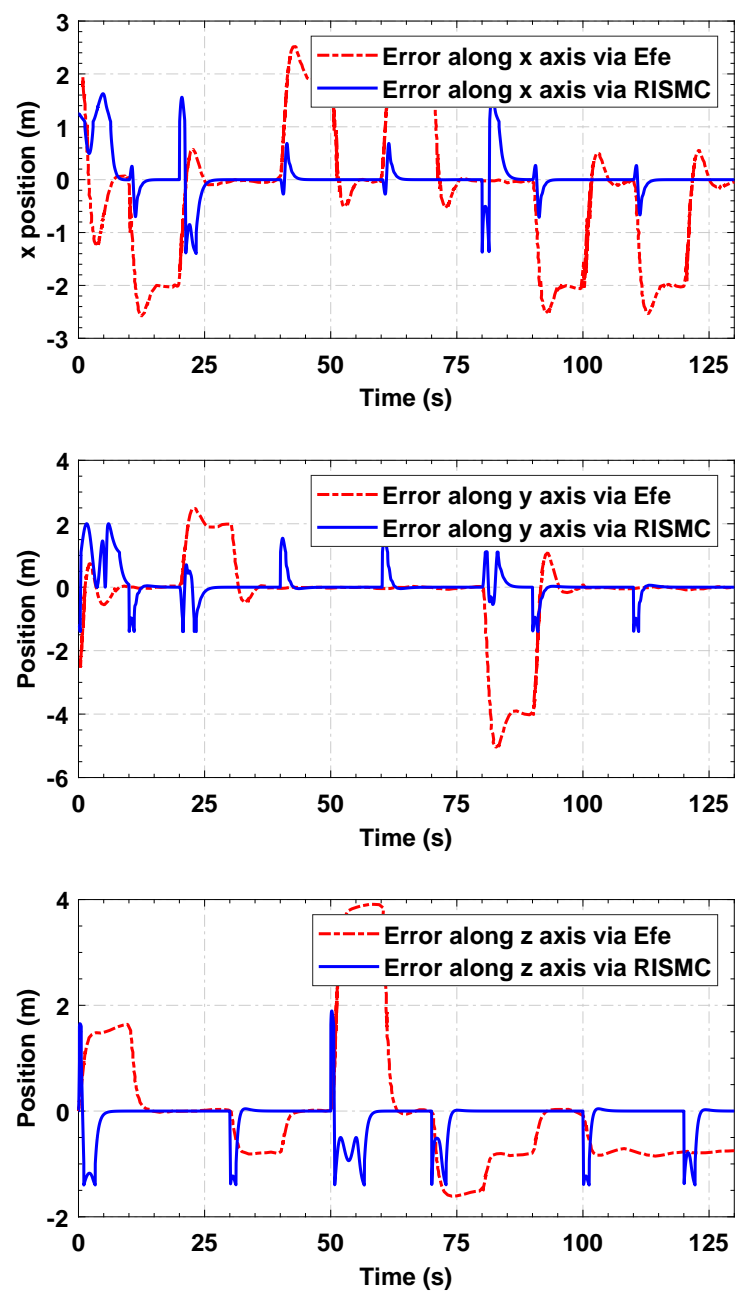

Fig. 3. Comparative errors regulation of the proposed RBISMC with standard literature [17]
In figure 3, a quite effective comparison is made in term of reference tracking errors (between the desired and real trajectories) along $x, y$ and $z$ axis. In addition, the comparative performances in term of regulation for the Euler angles i.e., roll, pitch and yaw, are shown in figure 4. The proposed RBISMC control law significantly decreases the overshoot and settling time with zero steadystate error. Note that the convergence for these Euler angles to the equilibrium is much better as compared to the standard results [17]. It is also clear that the proposed control scheme steers the corresponding tracking errors (along $x, y$ and $z$ ) and Euler angles $(\varphi, \vartheta, \psi)$ to their equilibrium values without having chattering problem, which makes the proposed control scheme more appealing as compared to FOISMC presented in [17]. Besides these advantages, the performance of the RBISMC controller is robust against the unwanted matched uncertainties $\Delta_{z}=0.3 \sin \left(z_{1}\right)+z_{1} z_{2}$ and $\Delta_{\psi}=0.1 \sin \left(\psi_{1}\right)+\psi_{1} \psi_{2}$ from the very start of the process.
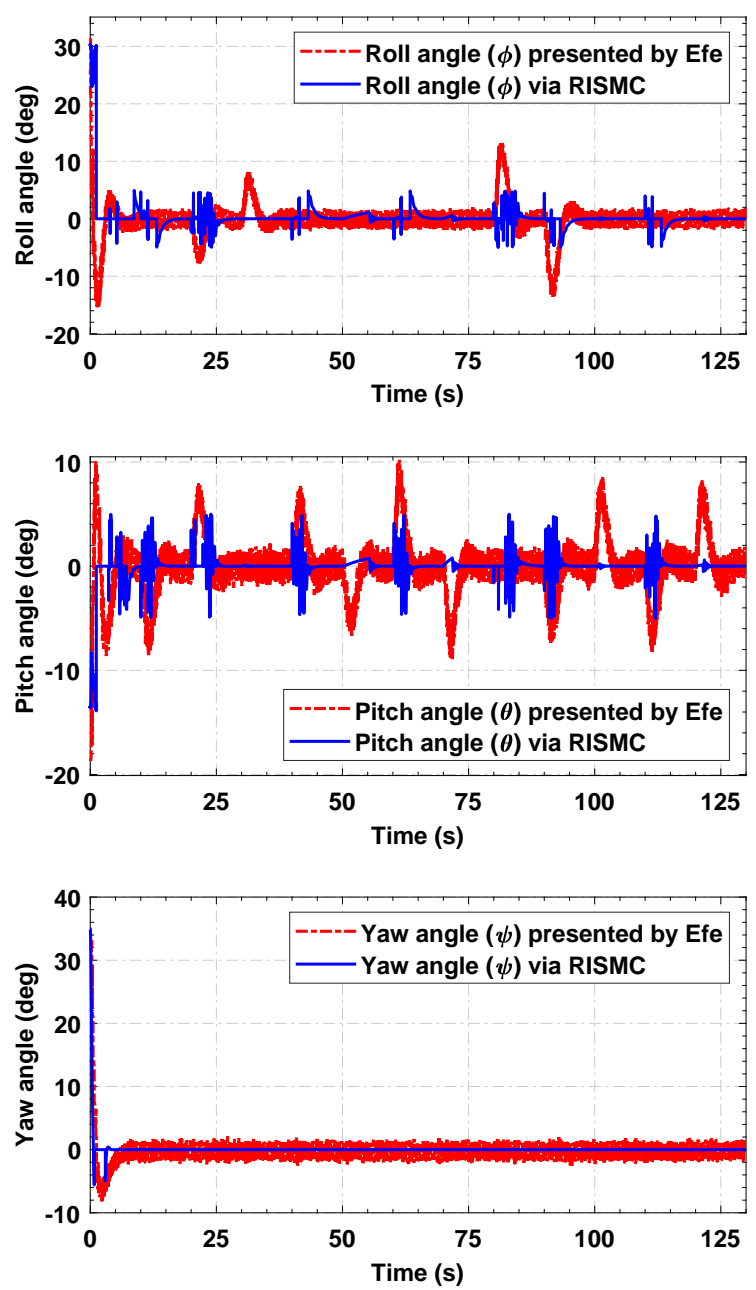

Fig. 4. The comparison of Euler angles trajectories of RBISMC with the standard literature [17] 

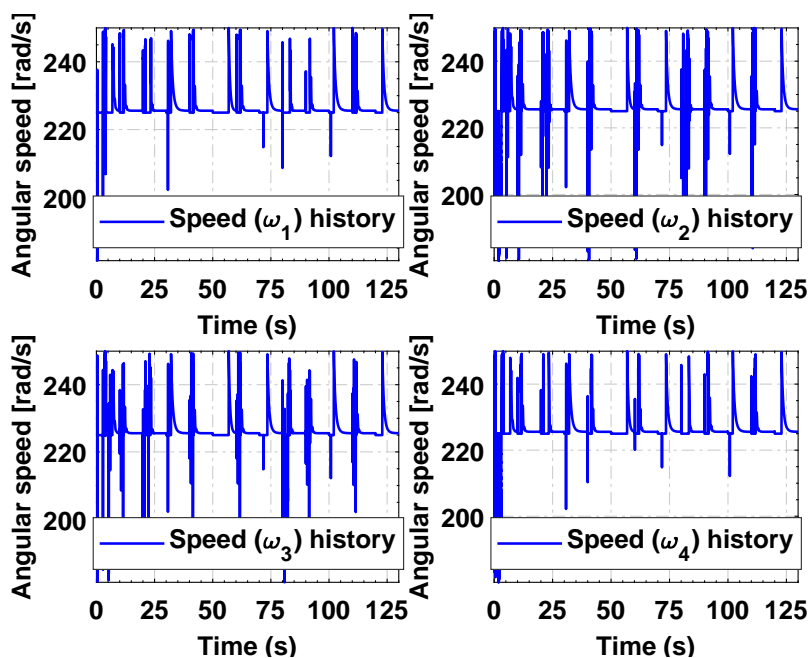

Fig. 5. History of control inputs in term of speeds

In figure 5, the control inputs of the proposed control scheme (required for fulfilling the main objective) are almost chatter and oscillations free whereas the counterpart presented oscillations with a considerable magnitude. The oscillations are dangerous for the system's health and consequently, degrades the dynamics' performance. The initial values of control inputs and angular velocities of the system are $\mathcal{U}_{l 0}=0(l=1,2,3,4)$ and $\dot{\varphi}=\dot{\vartheta}=\dot{\psi}=0$. The controller parameters $\kappa_{\chi_{1}}, \kappa_{\chi_{2}}, \kappa_{\theta_{1}}, \kappa_{\theta_{2}}, \kappa_{\psi_{1}}, \kappa_{\psi_{2}}, \kappa_{z_{1}}$ and $\kappa_{z_{2}}$, are selected as to force the system's states to the sliding surface. The parameters $\kappa_{\chi_{3}}, \kappa_{\theta_{3}}, \kappa_{\psi_{3}}$ and $\kappa_{z_{3}}$ are chosen to ensure robustness in the system against matched uncertainties.

\section{CONCLUSION}

The mathematical model, based on the EulerNewtonian and Euler-Lagrangian equations, is comprehensively presented in this work. For the control design, the overall system is subdivided into fully-actuated and under-actuated subsystems. The design of control law has been simplified with the effective combination of backstepping and ISMC based strategies, mainly by capitalizing the positive characteristics of both the nonlinear control techniques during the full flight maneuver of the quadcopter The overall closed loop stability is presented step by step for all the subsystems. The proposed control scheme claims improved robustness by eliminating the sensitive reaching phase of the conventional sliding modes. In addition, the chattering phenomena is alleviated by the proposed control law. A detailed simulation study is carried out and the obtained results are compared with the results from standard literature. It is, therefore, concluded that the proposed design is very effective for such kind of complex electro-mechanical systems.

\section{REFERENCES}

[1] Z. Ma, H. Li, Y. Gu, Z. Li, and Q. Li, "Flight and hover control system design for a mini-quadrotor based on multisensors," International Journal of Control, Automation and Systems, vol. 17, no. 2, pp. 486-499, February 2019.

[2] Y. Díaz-Méndez, M. S. de Sousa, G. Gomes, S. Cunha, and A. Ramos, "Analytical design and stability analysis of the universal integral regulator applied in flight control," International Journal of Control, Automation and Systems, vol. 17, no. 2, pp. 391-404, February 2019.

[3] M. Wasim, M. Ullah, and J. Iqbal, "Gain-scheduled proportional integral derivative control of taxi model of unmanned aerial vehicles," Revue Roumaine des Sciences TechniquesSerie Electrotechnique et Energetique, vol. 64, no. 1, pp. 75-80, January 2019.

[4] I. D. Cowling, O. A. Yakimenko, J. F. Whidborne, and A. K. Cooke, "A prototype of an autonomous controller for a quadrotor UAV," in European Control Conference (ECC). IEEE, 2007, pp. 4001-4008.

[5] S. A. Ajwad, J. Iqbal, R. U. Islam, A. Alsheikhy, A. Almeshal, and A. Mehmood, "Optimal and robust control of multi DOF robotic manipulator: Design and hardware realization," Cybernetics and Systems, pp. 1-17, January 2018.

[6] S. Bouabdallah and R. Y. Siegwart, "Full control of a quadrotor," in International Conference on Intelligent Robots and Systems, IROS 2007; Oct. 29, -Nov. 2, 2007, San Diego, CA. IEEE, 2007, pp. 153-158.

[7] X. Shi, Y. Cheng, C. Yin, S. Dadras, and X. Huang, "Design of fractional-order backstepping sliding mode control for quadrotor uav," Asian Journal of Control, vol. 21, no. 1, pp. 156-171, January 2019.

[8] W. Qi, G. Zong, and H. Karimi, "Sliding mode control for nonlinear stochastic singular semi-markov jump systems," IEEE Transactions on Automatic Control, May 2019.

[9] W. Qi, G. Zong, and H. R. Karim, "Observer-based adaptive smc for nonlinear uncertain singular semi-markov jump systems with applications to dc motor," IEEE Transactions on Circuits and Systems I: Regular Papers, vol. 65, no. 9, pp. 2951-2960, February 2018.

[10] F. Muñoz, E. S. Espinoza, I. González-Hernández, S. Salazar, and R. Lozano, "Robust trajectory tracking for unmanned aircraft systems using a nonsingular terminal modified super-twisting sliding mode controller," Journal of Intelligent \& Robotic Systems, vol. 93, no. 1-2, pp. 5572, February 2019.

[11] S.-L. Shi, J.-X. Li, and Y.-M. Fang, "Fractionaldisturbance-observer-based sliding mode control for fractional order system with matched and mismatched disturbances," International Journal of Control, Automation and Systems, vol. 17, no. 5, pp. 1184-1190, May 2019.

[12] D. Shi, Z. Wu, and W. Chou, "Super-twisting extended state observer and sliding mode controller for quadrotor uav attitude system in presence of wind gust and actuator faults," Electronics, vol. 7, no. 8, p. 128, August 2018.

[13] L. Luque-Vega, B. Castillo-Toledo, and A. G. Loukianov, "Robust block second order sliding mode control for a quadrotor," Journal of the Franklin Institute, vol. 349, no. 2, pp. 719-739, March 2012. 
[14] H. Sun, L. Hou, G. Zong, and X. Yu, "Fixed-time attitude tracking control for spacecraft with input quantization," IEEE Transactions on Aerospace and Electronic Systems, vol. 55, no. 1, pp. 124-134, June 2018.

[15] S. Irfan, A. Mehmood, M. T. Razzaq, and J. Iqbal, "Advanced sliding mode control techniques for inverted pendulum: Modelling and simulation," Engineering science and technology, an international journal, vol. 21, no. 4, pp. 753-759, August 2018.

[16] K. Nonaka and H. Sugizaki, "Integral sliding mode altitude control for a small model helicopter with ground effect compensation," in Proceedings of the 2011 american control conference. IEEE, 2011, pp. 202-207.

[17] M. Ö. Efe, "Integral sliding mode control of a quadrotor with fractional order reaching dynamics," Transactions of the Institute of Measurement and Control, vol. 33, no. 8, pp. 985-1003, December 2011.

[18] B. Samir, "Design and control of quadrotors with application to autonomous flying," Ecole Polytechnique federale de Lausanne, 2007.

[19] H.-P. Ren and X. Wang, "Experimental backstepping adaptive sliding mode control of hydraulic position servo system," in International Conference on Advanced Mechatronic Systems (ICAMechS). IEEE, December 2017, pp. 349-354.

[20] E. Suiçmez, "Trajectory tracking of a quadrotor unmanned aerial vehicle (uav) via attitude and position control," $E d u$ cational \& Industrial Television, July 2014. 